\title{
A hot spot in the neutrino flux from the Cygnus loop SNR
}

\author{
M. Bouyahiaoui, ${ }^{a, *}$ M. Kachelrie $\beta^{b}$ and D. V. Semikoz ${ }^{a}$ \\ ${ }^{a} A P C$, Université Paris Diderot, CNRS/IN2P3, CEA/IRFU, Observatoire de Paris, Sorbonne Paris Cité, \\ 11975205 Paris, France \\ ${ }^{b}$ Institutt for fysikk, NTNU, Trondheim, Norway
}

\begin{abstract}
A hot spot was found in the analysis of 7.5 years of data in the high-energy starting event sample of IceCube below the Galactic plane. We studied the cosmic rays density arround nearby, young supernova remnants assuming anisotropic diffusion. We then computed neutrino and gamma ray fluxes, combining cosmic rays densities with the distribution of matter in the nearby interstellar medium from a recent dust map survey. Cosmic rays from the cygnus loop produce a hot spot in neutrino close to the IceCube hotspot and with an intensity corresponding to two neutrino events.
\end{abstract}

37th International Cosmic Ray Conference -ICRC2021-

July 12th - July 23th, 2021

Berlin, Germany.

\footnotetext{
*Presenter
} 


\section{Introduction}

The IceCube collaboration has reanalyzed 7.5 years of data using neutrinos which interaction vertices are contained inside the fiducial volume of the detector in Ref. [1]. The energy spectrum of these "HESE" neutrinos is compatible with an unbroken power law $\mathrm{d} N / \mathrm{d} E \propto E^{-\alpha}$ with a spectral index of $\alpha=2.87 \pm_{0.19}^{0.20}$.

Gamma rays and neutrino spectra are correlated in hadronic interaction, thus estimates and strict upper bounds on neutrino flux can be deduced from gamma rays observations for specific cosmic rays sources, see e.g. Ref. [2].

In the case of young CR sources, the finite, energy-dependent distance CRs diffuse may lead, however, to a low-energy cutoff in the secondary spectrum. Therefore gamma-ray observations which are typically limited to energies $O(\mathrm{TeV})$ restrict only weakly the neutrino flux from such sources at $O(100 \mathrm{TeV})$. As result, the brightest spots in the Galactic neutrino sky may be gas clouds immersed into the $\mathrm{CR}$ overdensities close — but not close enough to be visible in $\mathrm{TeV}$ gamma-raysto nearby young CR sources [3].

Here, we report on a recent study [4] which focused the neutrino production by cosmic rays from nearby CR accelerators interacting with gas in the interstellar medium. In this work, we selected young and nearby sources and propagated cosmic rays from those supernova remnants (SNR), assuming anisotropic diffusion as suggested in Refs. [5-7].

The faster diffusion of CRs along magnetic field lines implies that gas clouds can give an important contribution to the secondary neutrino flux at larger distances than in the case of isotropic diffusion, if their perpendicular distance to the magnetic field line through the CR source is small. In addition, the slow diffusion perpendicular to the magnetic field enhances the CR density close to magnetic field lines connected to the CR sources. We extract the local matter distribution acting as target for neutrino production from extinction maps derived in Refs. [8, 9]. Combining then this matter map with the calculated CR densities, we find two prominent hot spots: One, produced by CRs from Vela is absent in the IceCube HESE sample. The other one, produced by CRs from the Cygnus Loop supernova remnant (SNR) is close to the most significant point in the IceCube search for point sources. We estimate the neutrino intensity of this hot spot and find it compatible with two observed events with energy above $60 \mathrm{TeV}$.

\section{Local cosmic ray sources}

We studied supernova remnants younger than $30 \mathrm{kyr}$ and located closer than $1 \mathrm{kpc}$ from the Sun ${ }^{1}$. We calculated CR density using same approach as in Refs. [10,11]. For the magnetic field, we approximated the Galactic magnetic field by the Jansson-Farrar model [12, 13], with a reduced turbulent field as suggested in Refs. [5-7]. Moreover, we modified this model as described in Ref. [11] to take into account the Local Bubble.

We employ the same injection spectrum for all sources for which we choose motivated by the results of Ref. [11] a broken power law in rigidity $\mathcal{R}=E /(Z e)$ with a break at $\mathcal{R}_{\mathrm{br}}=2 \times 10^{15} \mathrm{~V}$

\footnotetext{
${ }^{1}$ Data extracted from http://snrcat. physics . umanitoba.ca/SNRtable.php.
} 
and an exponential cut-off at $\mathcal{R}_{\max }=8 \times 10^{15} \mathrm{~V}$,

$$
\frac{d N_{i}}{d \mathcal{R}}=\left\{\begin{array}{lll}
N_{i} \mathcal{R}^{-2.2}, & \text { if } \quad \mathcal{R}<\mathcal{R}_{\mathrm{br}} \\
\tilde{N}_{i} \mathcal{R}^{-3.1} \exp \left(-\mathcal{R} / \mathcal{R}_{\max }\right), & \text { if } \quad \mathcal{R} \geq \mathcal{R}_{\mathrm{br}} .
\end{array}\right.
$$

We inject 5.000 protons per energy at the position of each source and propagate them for the time $\tau$ in our magnetic field model. Then we calculate the CR density $n(E)$ in each cell of size $(6 \mathrm{pc})^{3}$. The energy injected in CRs by each source is chosen as $3.5 \times 10^{50} \mathrm{erg}$ in protons and $2.6 \times 10^{50} \mathrm{erg}$ in helium, respectively, which is compatible with the expectation that $\simeq 50-60 \%$ of the explosion energy are transferred into relativistic particles in the case of efficient CR accelerators [14-16].

\section{Local matter density from dust maps}

The interstellar medium (ISM) is composed by dust and gas. Even if dust contributes only a small mass fraction of the total mass, it efficiently absorbs and scatters photons with wavelengths in the visible and ultraviolet range. Therefore the distribution of dust can be used as a tracer of, e.g., gas which serves CRs as target for secondary neutrino and gamma-ray production.

The authors of Refs. [8, 9] realised a study on the closer neighborhood of the Sun, building a dust map for a $740^{2} \times 540 \mathrm{pc}^{3}$ cube with a resolution of $1 \mathrm{pc}$. Using Gaia, 2MASS, PANSTARRS, and ALLWISE data, they deduced the $\mathrm{G}$ band extinction of five million stars with known parallaxes. Their results for the 3D distribution of dust are publicly available as a grid containing the e-folds of extinction per cell.

The extinction due to dust is proportional to the hydrogen column density along the grid cell as [17]

$$
N_{\mathrm{H}}=2.87 \times 10^{21} \mathrm{~cm}^{-2} A_{\mathrm{V}} / \mathrm{mag} .
$$

Using moreover $A_{\mathrm{G}} / A_{\mathrm{V}}=0.789$ as the selective extinction in the GAIA G band from Ref. [18], we obtain

$$
N_{\mathrm{H}}=3.63 \times 10^{21} \mathrm{~cm}^{-2} A_{\mathrm{G}} / \mathrm{mag} .
$$

In addition, we account for helium which contributes $9.1 \%$ to the number density of the ISM.

\section{Neutrino intensity}

We calculated the neutrinos produced by CRs from SNR G074.0-08.5 in the Cygnus Loop. The intensity $I_{v}(E, l, b)$ of neutrinos with energy $E$ emitted along the line-of-sight with direction $(l, b)$ is given by

$$
I_{v}(E, l, b)=\sum_{A, A^{\prime}} \int_{E}^{\infty} \mathrm{d} E^{\prime} \int_{0}^{\infty} \mathrm{d} s n_{\mathrm{gas}}^{A}(\boldsymbol{x}) I_{C R}^{A}\left(E^{\prime}, \boldsymbol{x}\right) \frac{\mathrm{d} \sigma^{A A^{\prime} \rightarrow v}\left(E^{\prime}, E\right)}{\mathrm{d} E} .
$$

We include both for the target gas and the CRs the contribution of protons and helium nuclei, $A, A^{\prime}=\{p, \mathrm{He}\}$. The neutrino production cross sections are evaluated with AAfrag [19]. The maximum of $I_{v}(E, \alpha, \delta)$ corresponds to $\simeq 400$ times the isotropic neutrino intensity measured by IceCube. 


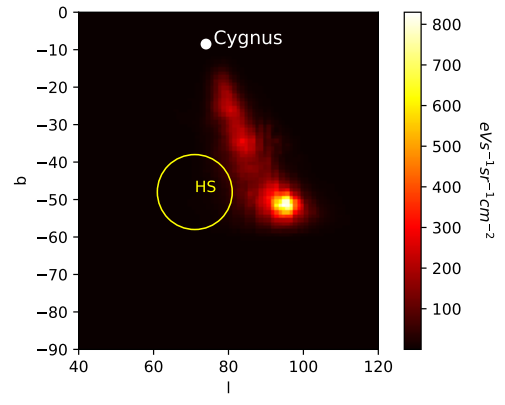

Figure 1: Neutrino intensity $E^{2} I_{\nu}(E, \alpha, \delta)$ for $E=100 \mathrm{TeV}$ in equatorial coordinates; the star shows the most significant point in the IceCube search for point sources.

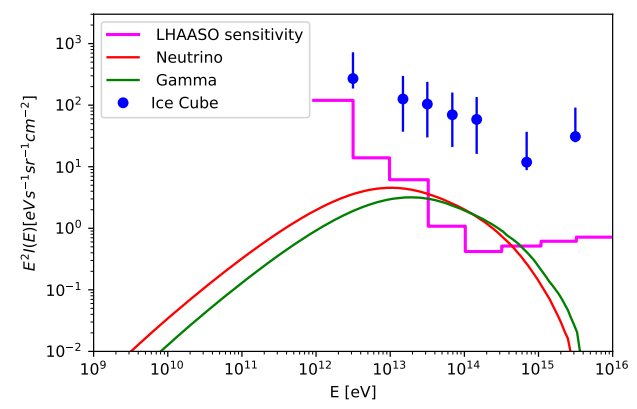

Figure 2: Equivalent isotropic intensity of a neutrinos and photons produced by CRs from the Cygnus Loop are compared to IceCube data [20] and the estimated sensitivity of the LHASSO experiment [21].

We define the equivalent isotropic intensity of a specific source as

$$
I_{v}(E) \equiv \frac{1}{4 \pi} \int \mathrm{d} \Omega w(E) I_{v}(E, l, b)
$$

with the weight $w(E)$ set to one. Since the observed neutrino intensity $I_{v}^{\mathrm{obs}}(E)$ is approximately isotropic, the ratio $I_{v}(E) / I_{v}^{\mathrm{obs}}(E)$ corresponds to the fraction of neutrino events with energy $E$ from a given source observed by an experiment with uniform exposure. In Fig. 2, we show the isotropic neutrino intensity $I_{v}(E)$ produced by CRs from the Cygnus Loop together with Ice Cube data from Ref. [20]. Note that the neutrino flux is dominated by the contribution from helium primaries which have a larger interaction probability and dominate above $E \simeq 10^{14} \mathrm{eV}$ the $\mathrm{CR}$ injection spectrum defined in Eq. (1). Additionally, we compare the intensity of photons to the sensitivity of the LHASSO experiment estimated in Ref. [21].

For a specific experiment like IceCube, we have to account for the declination dependence of the effective area $A_{\mathrm{eff}}(E, \delta)$. For the HESE data set, we use the the effective area $A_{\mathrm{eff}}(E)$ from Ref. [1] and deduce the declination dependence from Ref. [22]. Since the extension of the hot spot is small, we can neglect the declination dependence of the weight, setting $w=A_{\text {eff }}\left(E, \delta_{s}\right) / A_{\text {eff }}(E) \simeq 1$ with $\delta \simeq 0^{\circ}$ for the hot spot of IceCube.

We estimate the number of expected neutrino events above the minimal energy $E_{0}$ as

$$
N_{v}\left(E>E_{0}\right)=w T \int_{E_{0}}^{\infty} d E \int \mathrm{d} \Omega A_{\mathrm{eff}}(E) I_{v}(E, l, b),
$$

where $T$ is the observation time. With $E_{0}=60 \mathrm{TeV}$, we find that the hot spot produced by CRs from the Cygnus loop corresponds to two neutrino events, while four neutrino events in the HESE (event ID 44, 67, 74 with corresponding energies of $84.6 \mathrm{TeV}, 165 \mathrm{TeV}, 71.3 \mathrm{TeV}$ respectively, and event 105) may be associated with hot spot found in the IceCube analysis.

Before we conclude, we comment on some peculiarities of our model. First, we note that the position of the hot spot is approximately determined by the intersection of the ellipsoid filled by CRs and the boundary of the Local Bubble. From Fig. 1, we see that the hot spot predicted by us 


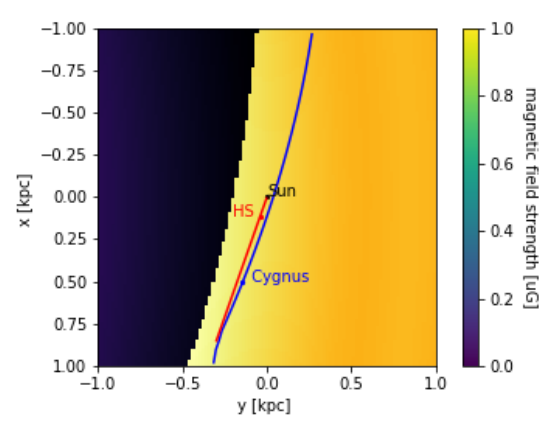

(a) XY projection

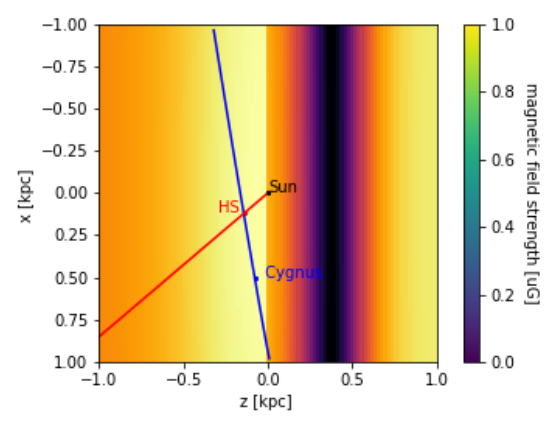

(b) $\mathrm{XZ}$ projection

Figure 3: Projection of the magnetic field line passing through the Cygnus loop SNR in blue, the position of the Sun position in black and the red line represent the position of the IceCube hot spot for varying $R$, the red point corresponds to the position of the IceCube hot spot for $R_{h s}=194 \mathrm{pc}$.

is around $10^{\circ}-15^{\circ}$ offset against the most significant point in the search for point sources found by IceCube. Uncertainties in the direction of the magnetic field line connected to the Cygnus SNR lead to a variation in the position of the hot spot as shown in Fig. 3: In this figure, we show the projection of the magnetic field line passing through Cygnus (blue line) together with the position of IceCube hot spot varying its distance $R$ as a red line. If this hot spot is created by the cosmic rays from the Cygnus SNR, its distance $R$ has to be around $200 \mathrm{pc}$ such that the IceCube hot spot is the closest to CR ellipsoid from Cygnus. From Fig. $3 \mathrm{a}$, it is clear that a rather small rotation of $\simeq 5^{\circ}$ of the magnetic field line in the Galactic plane would be sufficient to align cosmic rays from the Cygnus loop SNR with the position of the Ice Cube hot spot. Note also that small changes in the position of the hot spot would not lead to large changes in the resulting neutrino intensity, because the gas density in the hot spot is not atypically large, $n_{\text {gas }} \simeq 0.6 / \mathrm{cm}^{3}$, compared to other, close parts of the boundary of the Local Bubble.

\section{Conclusions}

We have proposed in this work that CRs from the SNR G074.0-08.5 in the Cygnus Loop interacting with gas close to the boundary of the Local Bubble lead to an hot spot in the neutrino flux. Anisotropic diffusion of CRs is a necessary ingredient of this proposals, leading to enhanced CR densities along magnetic field lines connected to CR sources. The position of this hot spot is compatible with the most significant point in the IceCube search for point sources, and the neutrino intensity estimated by us corresponds to two events with energy above $60 \mathrm{TeV}$. The corresponding photon fluxes should be detectable by LHASSO within few years, providing a clear signature for a Galactic origin of the hot spot in the neutrino flux observed by IceCube.

\section{References}

[1] ICECube collaboration, R. Abbasi et al., The IceCube high-energy starting event sample: Description and flux characterization with 7.5 years of data, 2011.03545. 
[2] F. L. Villante and F. Vissani, How precisely neutrino emission from supernova remnants can be constrained by gamma ray observations?, Phys. Rev. D 78 (2008) 103007 [0807 . 4151].

[3] M. Kachelrießand S. Ostapchenko, Neutrino yield from Galactic cosmic rays, Phys. Rev. D90 (2014) 083002 [1405 . 3797].

[4] M. Bouyahiaoui, M. Kachelriess and D. V. Semikoz, A hot spot in the neutrino flux created by cosmic rays from the Cygnus Loop, 2105.13378.

[5] G. Giacinti, M. Kachelrieß and D. V. Semikoz, Explaining the Spectra of Cosmic Ray Groups above the Knee by Escape from the Galaxy, Phys. Rev. D90 (2014) 041302 [1403. 3380].

[6] G. Giacinti, M. Kachelrieß and D. V. Semikoz, Escape model for Galactic cosmic rays and an early extragalactic transition, Phys. Rev. D91 (2015) 083009 [1502 . 01608].

[7] G. Giacinti, M. Kachelrieß and D. V. Semikoz, Reconciling cosmic ray diffusion with Galactic magnetic field models, JCAP 1807 (2018) 051 [1710.08205].

[8] R. H. Leike and T. A. Enßlin, Charting nearby dust clouds using gaia data only, Astron. Astrophys. 631 (2019) A32.

[9] R. H. Leike, M. Glatzle and T. A. Enßlin, Resolving nearby dust clouds, Astron. Astrophys. 639 (2020) A138.

[10] M. Bouyahiaoui, M. Kachelrieß and D. V. Semikoz, Vela as the Source of Galactic Cosmic Rays above $100 \mathrm{TeV}$, JCAP 1901 (2019) 046 [1812.03522].

[11] M. Bouyahiaoui, M. Kachelrieß and D. V. Semikoz, High-energy neutrinos from cosmic ray interactions in the Local Bubble, Phys. Rev. D 101 (2020) 123023 [2001.00768].

[12] R. Jansson and G. R. Farrar, The Galactic Magnetic Field, Astrophys.J. 761 (2012) L11 [1210.7820].

[13] R. Jansson and G. R. Farrar, A New Model of the Galactic Magnetic Field, Astrophys.J. 757 (2012) 14 [1204.3662].

[14] A. R. Bell and S. G. Lucek, Cosmic ray acceleration to very high energy through the non-linear amplification by cosmic rays of the seed magnetic field, Mon. Not. Roy. Astron. Soc. 321 (2001) 433.

[15] D. C. Ellison, A. Decourchelle and J. Ballet, Hydrodynamic simulation of supernova remnants including efficient particle acceleration, Astron. Astrophys. 413 (2004) 189 [astro-ph/0308308].

[16] A. R. Bell, Turbulent amplification of magnetic field and diffusive shock acceleration of cosmic rays, Mon. Not. Roy. Astron. Soc. 353 (2004) 550.

[17] D. R. Foight, T. Güver, F. Özel and P. O. Slane, Probing X-Ray Absorption and Optical Extinction in the Interstellar Medium Using Chandra Observations of Supernova Remnants, Astrophys. J. 826 (2016) 66 [1504.07274]. 
[18] S. Wang and X. Chen, The Optical to Mid-infrared Extinction Law Based on the APOGEE, Gaia DR2, Pan-STARRS1, SDSS, APASS, 2MASS, and WISE Surveys, Astrophys. J. 877 (2019) 116 [1904.04575].

[19] M. Kachelrieß, I. V. Moskalenko and S. Ostapchenko, AAfrag: Interpolation routines for Monte Carlo results on secondary production in proton-proton, proton-nucleus and nucleus-nucleus interactions, Comput. Phys. Commun. 245 (2019) 106846 [1904.05129].

[20] IcECube collaboration, M. G. Aartsen et al., Constraints on Galactic Neutrino Emission with Seven Years of IceCube Data, Astrophys. J. 849 (2017) 67 [1707. 03416].

[21] A. Neronov and D. Semikoz, LHAASO telescope sensitivity to diffuse gamma-ray signals from the Galaxy, Phys. Rev. D 102 (2020) 043025 [2001.11881].

[22] IceCube collaboration, M. G. Aartsen et al., Observation of High-Energy Astrophysical Neutrinos in Three Years of IceCube Data, Phys. Rev. Lett. 113 (2014) 101101 [1405 . 5303]. 